\title{
Editorial Note: Ocular leprosy; theme for 1984
}

This number carries an article on leprosy and the eye, 'A computer form to aid in the collection of data on the ocular complications of leprosy' by Mr T J ffytche. At a recent meeting of the Editorial Board it was decided to publish further articles on the theme of ocular leprosy during 1984. Contributions on this subject, whether as original articles, case reports, or letters will be most welcome. 